\title{
Time and Frequency Response of the Conventional Avalanche Photodiode
}

\author{
MALVIN C. TEICH, SENIOR MEMBER, IEEE, KUNIAKI MATSUO, MEMBER, IEEE, \\ AND BAHAA E. A. SALEH, SENIOR MEMBER, IEEE
}

\begin{abstract}
An analytical expression for the time course of the average impulse response function for a conventional avalanche photodiode is derived. Delta-function absorption of a single photocarrier and single-carrier-initiated/single-carrier multiplication conditions are assumed. The result is obtained as a limiting case of a previously derived equation for the staircase avalanche photodiode. The initial exponential growth of the curves is shown to represent electron and hole contributions arising from multiplication in the avalanche region whereas the subsequent exponential decay arises from residual holes transiting backward across the multiplication region. The associated frequency response function is obtained by Fourier transformation. The analytical results are shown to be in good accord with average impulse response functions obtained by Riad and Hayes by means of simulation from the transport equations. The results should also apply to the channeling avalanche photodiode and to related structures in which the carriers are spatially separated and the multiplication is essentially single-carrier like.
\end{abstract}

\section{INTRODUCTION}

$\mathrm{T}$ HE USEFULNESS of avalanche photodiodes (APD's) as detectors in fiber-optic communication systems has been well established [1]. These devices operate by converting clusters of detected photons, associated with information-carrying pulses of light in a digital communication system, into cascades of electrons. These electron cascades have sufficiently high charge to be detected by the electronic circuitry following the APD. The avalanche multiplication process introduces noise because the number of electrons created per detected photon varies from trial to trial. The noise properties, which in large part determine the sensitivity of the optical receiver, have been studied for many different kinds of APD's [2]-[13]. These studies generally invoke the assumption of instantaneous multiplication, meaning that the detector integration time is sufficiently long so that the entire current pulse is captured as a charge.

Aside from receiver sensitivity, it is important to know

Manuscript received February 5, 1986; revised June 4, 1986. This work was supported by the National Science Foundation and by the Joint Services Electronics Program.

M. C. Teich is with the Center for Telecommunications Research, Department of Electrical Engineering, Columbia University, New York, NY 10027.

K. Matsuo was with the Columbia Radiation Laboratory, Department of Electrical Engineering, Columbia University, New York, NY 10027. He is now with the Hiroshima-Denki Institute of Technology, Hiroshima, Japan.

B. E. A. Saleh is with the Department of Electrical and Computer Engineering, University of Wisconsin, Madison, WI 53706.

IEEE Log Number 8610061. the maximum bit rate at which a fiber-optic communication system can operate. This is determined in part by how quickly the APD current decays in response to a pulse of photons. Only then can the device receive the next bit without having to be concerned about the presence of residual current from the previous bit (intersymbol interference) [1]. The time course of the current produced in the APD circuit arises from several sources [2] (devices are usually constructed in such a way that the diffusion current can be ignored). The current component arising from the motion of carriers through the region where avalanche multiplication takes place has a duration at least as long as the time that it takes the carrier pairs (electrons and holes) to complete their drift through this region. The transit time through the avalanche multiplication region is designated $\tau_{m}$. The shape of this average current pulse is determined both by the average multiplication $\langle M\rangle$ and by the electron and hole velocities, whereas its area (charge) is determined solely by the average multiplication. Furthermore, if electrons and holes are both capable of causing impact ionization in the multiplication region, the resulting feedback process can lead to the persistance of carriers in this region, and an associated increase in the duration of the current pulse. This additional time is called the avalanche buildup time $\tau_{a v}[14]$; it is dependent on the width of the multiplication region $w$, and on $\langle M\rangle, \tau_{m}$, and the ratio of ionization coefficients for holes and electrons $k_{c}=\beta / \alpha[15]$. For single-carrier multiplication, $\tau_{a v}=0$. Even for double-carrier multiplication, at usual values of the gain, $\tau_{a v}$ may often be neglected in comparison with the transit time through the multiplication region $\tau_{m}$ [15].

In many device structures, such as the reach-through APD (RAPD) [16] and the separate-absorption-gradingmultiplication region (SAGM) APD [17], the depletion region extends well beyond the multiplication region. In such devices the APD current pulse is further lengthened by the transit time through this nonmultiplying depletion region $\tau_{t r}$. Indeed $\tau_{t r}$ is often the limiting factor that determines the APD response time [7]. Of course, the receiver circuitry to which the APD is connected (associated with the response time $\tau_{R C}$ ) also plays a role in determining the speed of response of the system. However, in the current state of our engineering expertise this is not often the limiting factor.

In this paper we derive an analytical expression for the time course of the average current pulse arising from a 
single photocarrier entering the multiplication region of a conventional APD (CAPD), assuming single-carrier-initiated/single-carrier multiplication (SCISCM). ${ }^{1}$ This is designated the single-photocarrier average impulse response function $\langle h(t)\rangle$. The full-width duration of $\langle h(t)\rangle$ is $\tau_{m}$ since $\tau_{a v}=0$ as a result of the SCISCM assumption. The result is obtained by carrying to the limit (of an infinite number of stages) the recently-derived expression for $\langle h(t)\rangle$ for the staircase avalanche photodiode [11]. The underlying model for our calculations is the Bernot.1li branching process that, in the limit of an infinite number of stages and vanishingly small multiplication per stage, goes over to the pure birth process with linear birth rite [18]. Delta-function absorption is assumed at the bourdary of the multiplication region. An analytical result for the associated average frequency response function $H(j \omega)$ is obtained by Fourier transformation.

Over the years, there have been a great many calcu ations of various APD frequency response functions. Almost all of these have made use of the carrier transpirt equations as a point of departure. Indeed, many of the results have sufficient generality to account for doubecarrier multiplication with unequal ionization coefficients and unequal carrier velocities [19]-[24]. Although there is a formal equivalence between the average frequency response function $H(j \omega)$ and the average impulse response function $\langle h(t)\rangle$, direct calculations of the latter $c$ re often of substantial interest because they are solutions of the time-domain stochastic differential equations describing carrier transport in the multiplication region [25]. Orly a handful of explicit results for the average impulse response function have appeared, namely the analytical calculations of Ruegg [16] and Walma and Hackam [26], and the simulation results of Riad and Hayes [27] and Ri and Riad [25]. Because our CAPD device model is the same as that used for single-carrier simulation results by Riad and Hayes, we will find it useful to compare our analytical results with their computer calculations.

\section{Impulse Response Function}

The time course of the current pulse arising from a single carrier entering the multiplication region of an AF'D is a superposition of the contributions from both the initial carrier itself and from the offspring carrier pairs. The 1esult for the CAPD can be most easily understood in terms of the discrete current contributions in the staircase AFD with deterministic transit time [11, Section III, Fig. (i]. The staircase APD is a graded-gap superlattice device designed in such a way that only electrons are expected to impact ionize [9], [28], [29]. It is one of a family of superlattice avalanche photodiodes (SAPD's) that may find use in fiber-optic communication systems operating in the

\footnotetext{
${ }^{1}$ The designation single-carrier-initiated/single-carrier multiplication (SCISCM) means that only a single kind of carrier (viz., either electrcns or holes) initiates the avalanche process and that only this kind of carr er is capable of creating new carrier pairs by impact ionization. In addition we have assumed that only a single photocarrier is injected, meaning that only one electron (or hole) is injected.
}

1.3-1.6 $\mu \mathrm{m}$ wavelength region [30], [31]. ${ }^{2}$ In principle, structures of this kind can eliminate the feedback noise associated with two-carrier behavior [11], but in practice the effects of residual hole ionization may not be able to be entirely eliminated [12], [29].

Nevertheless, the single-carrier staircase APD impulse response functions derived in [11] provides a useful point of departure for obtaining the CAPD impulse response function. This is because the behavior of the staircase APD becomes identical to that of the single-carrier CAPD [11]-[13], [18] in the limit of an infinite number of stages and vanishingly small multiplication per stage. Formally, the staircase-device current can be viewed as a marked filtered Bernoulli branching process.

The impulse response function $h(t)$ is of course random [11, Eq. (13)]. Our interest at this point is in the average single-photocarrier impulse response function for the $m$ stage staircase APD with ionization probability $P$, which may be written as [11, Eq. (14)]

$$
\begin{aligned}
\left\langle h^{(m)}(t)\right\rangle= & \left\langle a_{0}^{(m)}(t)\right\rangle \\
& +P \sum_{k=1}^{m}(1+P)^{k-1}\left[\left\langle a_{k}^{(m)}(t)\right\rangle+\left\langle b_{k}^{(m)}(t)\right\rangle\right] .
\end{aligned}
$$

The current pulses produced by electrons and holes created at the $k$ th step are designated $\left\langle a_{k}^{(m)}(t)\right\rangle$ and $\left\langle b_{k}^{(m)}(t)\right\rangle$, respectively. These are given by [11, Fig. 6]

$$
\begin{aligned}
\left\langle a_{k}^{(m)}(t)\right\rangle= & {\left[q /(m+1) \tau_{e}\right]\left[U\left(t-k \tau_{e}\right)\right.} \\
& \left.-U\left(t-(m+1) \tau_{e}\right)\right] \\
\left\langle b_{k}^{(m)}(t)\right\rangle= & {\left[q /(m+1) \tau_{h}\right]\left[U\left(t-k \tau_{e}\right)\right.} \\
& \left.-U\left(t-k\left(\tau_{e}+\tau_{h}\right)\right)\right]
\end{aligned}
$$

where the symbol $q$ represents the electronic charge, $U$ is the unit step function, and $\tau_{e}$ and $\tau_{h}$ are the (deterministic) electron and hole transit times through a single graded region, respectively. The impulse response function is comprised of a superposition of electron and hole currents which, in turn, are weighted sums of the $a_{k}$ 's and the $b_{k}$ 's. Expressions can also be obtained for higher moments and for the autocorrelation function of the impulse response, but the procedure rapidly becomes quite lengthy. These

\footnotetext{
${ }^{2} \mathrm{~A}$ number of novel superlattice devices have been proposed for enhancing the ionization-coefficient ratio and reducing tunneling currents. The first such proposal, by Chin et al. [32], suggested the use of a multiquantum-well SAPD consisting of an alternating series of wide- and narrowbandgap layers. It has been shown both experimentally [33]-[35], and by means of many-particle Monte Carlo simulations [29], that such a structure can indeed provide an enhanced ionization ratio. Other heterostructure configurations that have been considered include the doped-quantum-well SAPD conceived by Blauvelt et al. [36], [37] and the stored-carrier multiquantum-well SAPD of Smith et al. [38]-[40]. An effort is currently underway at AT\&T Bell Laboratories to fabricate a staircase SAPD for operation in the long wavelength region of interest for fiber-optic communications [41]. The excess noise factor, counting distributions, and system-performance characteristics for SAPD's in which the carrier transport is perpendicular to the superlattice planes have recently been obtained [11][13].
} 
quantities would be useful in a careful determination of the effects of intersymbol interference in an optical communication system.

The single-photocarrier average impulse response function for the CAPD, $\langle h(t)\rangle \equiv\left\langle h^{(\infty)}(t)\right\rangle$, is readily obtained by increasing the number of staircase stages $m$ without limit while simultaneously decreasing the transit times through each graded region $\tau_{e}$ and $\tau_{h}$. Since the transit times per stage will become vanishingly small, it is more convenient to refer to them as $\Delta \tau_{e}$ and $\Delta \tau_{h}$, respectively. Thus, we let

$$
\begin{aligned}
& m \rightarrow \infty \\
& \tau_{e} \equiv \Delta \tau_{e} \rightarrow 0 \\
& \tau_{h} \equiv \Delta \tau_{h} \rightarrow 0 .
\end{aligned}
$$

Simultaneously, the products $m \Delta \tau_{e}$ and $m \Delta \tau_{h}$ are kept constant such that

$$
\begin{aligned}
& m \Delta \tau_{e}=w / v_{e} \\
& m \Delta \tau_{h}=w / v_{h}
\end{aligned}
$$

where $w$ is the width of the multiplication region and $v_{e}$ and $v_{h}$ are the (deterministic) electron and hole velocities, respectively. In this limit the electron ionization probability $P$ (or $\Delta P$ ) can be written

$$
P \equiv \Delta P=\alpha \Delta x
$$

where $\alpha$ is the electron ionization coefficient and $\Delta x$ is the width of one graded region. Thus

$$
m \Delta P=\alpha w
$$

assuming constant electric field throughout the avalanche region.

Using (2)-(8), and letting $\Delta x \rightarrow 0,(1)$ becomes

$$
\begin{aligned}
\langle h(t)\rangle= & \frac{q v_{e}}{w}\left[U(t)-U\left(t-w / v_{e}\right)\right] \\
& +\frac{q v_{e}}{w} \alpha \int_{0}^{w} d x e^{\alpha x}\left[U\left(t-x / v_{e}\right)-U\left(t-w / v_{e}\right)\right] \\
& +\frac{q v_{h}}{w} \alpha \int_{0}^{w} d x e^{\alpha x}\left[U\left(t-x / v_{e}\right)-U\left(t-w / v_{\mu}\right)\right]
\end{aligned}
$$

where

$$
1 / v_{\mu}=1 / v_{e}+1 / v_{h}
$$

Performing the simple integrations in (9) leads to

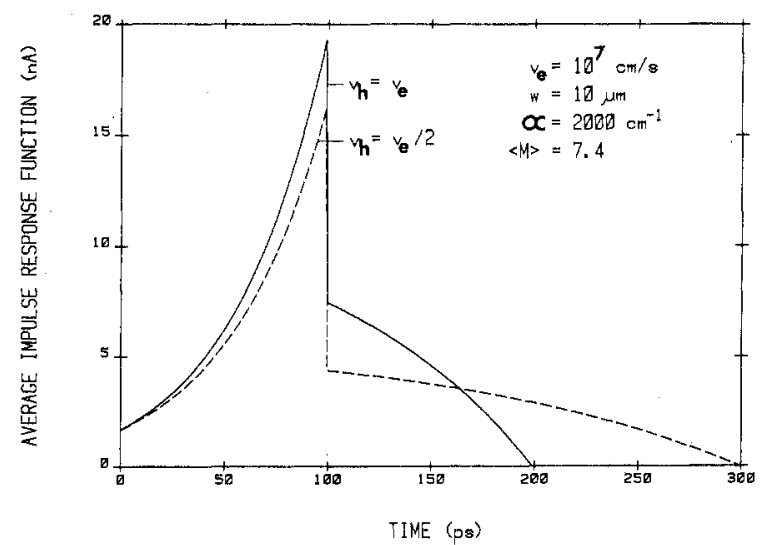

Fig. 1. Theoretical average impulse response function $\langle h(t)\rangle$ versus time $t$ for a single-carrier conventional avalanche photodiode with single-electron injection. For purposes of illustration, the parameter values were chosen as follows: electron velocity $v_{e}=10^{7} \mathrm{~cm} / \mathrm{s}$, width of multiplication region $w=10 \mu \mathrm{m}$, and electron ionization coefficient $\alpha=2000$ $\mathrm{cm}^{-1}$. Curves are shown for two values of hole velocity: $v_{h}=v_{e}$ (solid curve) and $v_{h}=v_{e} / 2$ (dashed curve). The ordinate is displayed in units of nanoamperes and the abscissa in units of picoseconds. The area under each curve provides the average gain $\langle M\rangle=\exp (\alpha w)=7.4$, in accordance with (12).

The integral of the average impulse response function is simply the total charge created by a single injected carrier, i.e.,

$$
\int_{-\infty}^{\infty}\langle h(t)\rangle d t=q \exp (\alpha w) .
$$

Graphical representations of the average impulse response function versus time given in (11) are presented in Figs. 1 and 2 for several representative sets of data with single-electron injection. The parameter values used in the two figures are similar except for the electron ionization coefficient $\alpha$, which has been set equal to $2000 \mathrm{~cm}^{-1}$ in Fig. 1 and $5000 \mathrm{~cm}^{-1}$ in Fig. 2. As a result, the ordinate in Fig. 1 is displayed in units of nanoamperes whereas the ordinate in Fig. 2 is in units of microamperes. A comparison of the $\langle h(t)\rangle$ curves in the two figures shows similar shapes although, of course, the gain is substantially higher in Fig. 2.

The initial exponential growth of the curves represents electron and hole contributions arising from multiplication in the avalanche region. Assuming that it is an electron that is injected, this continues for the entire time that it requires to travel across the multiplication region, i.e., $t_{e}=w / v_{e}$. The subsequent exponential decay then represents residual holes transiting backward across the multiplication region. These lead to a circuit current during an additional time $t_{h}=t_{e}$ when $v_{h}=v_{e}$, or time $t_{h}=2 t_{e}$ when $v_{h}=v_{e} / 2$.

$$
\langle h(t)\rangle= \begin{cases}\frac{q v_{e}}{w}\left\{\exp \left(\alpha v_{e} t\right)+\left[v_{h} / v_{e}\right]\left[\exp \left(\alpha v_{e} t\right)-\exp \left(\alpha v_{\mu} t\right)\right]\right\}, & 0 \leq t \leq w / v_{e} \\ \frac{q v_{h}}{w}\left[\exp (\alpha w)-\exp \left(\alpha v_{\mu} t\right)\right], & w / v_{e}<t \leq w / v_{\mu} \\ 0, & \text { elsewhere. }\end{cases}
$$




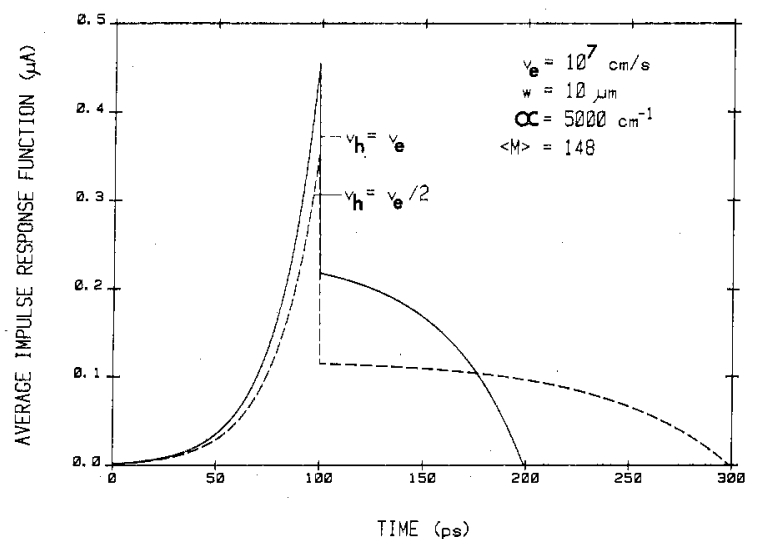

Fig. 2. Theoretical average impulse response function $\langle h(t)\rangle$ versus ti ne $t$ for a single-carrier conventional avalanche photodiode with single-el:sctron injection. For purposes of illustration, the parameter values were chosen as follows: electron velocity $v_{e}=10^{7} \mathrm{~cm} / \mathrm{s}$, width of multiflication region $w=10 \mu \mathrm{m}$, and electron ionization coefficient $\alpha=5000$ $\mathrm{cm}^{-1}$. Curves are shown for two values of hole velocity: $v_{h}=v_{e}$ (solid curve) and $v_{h}=v_{e} / 2$ (dashed curve). In this case, the ordinate is displayed in units of microamperes and the abscissa in units of picoseconds. The area under each curve provides the average gain $\langle M\rangle=\exp (\alpha w)$ $=148$. A comparison with the results in Fig. 1 shows a similar shape for $\langle h(t)\rangle$ but of course the gain is substantially higher.

\section{Frequency Response Function}

The average frequency response function for the CAP), $H(j \omega)$, is obtained as the Fourier transform of the average impulse response function given in (11). Although the result is somewhat lengthy, it is actually quite direct, viz.,

$$
\begin{aligned}
& H(j \omega)=\frac{a(1+\gamma)}{u^{2}+\omega^{2}}\left\{u\left[\exp \left(u t_{e}\right) \cos \left(\omega t_{e}\right)-1\right]\right. \\
& \left.+\omega \exp \left(u t_{e}\right) \sin \left(\omega t_{e}\right)\right\} \\
& -\frac{a \gamma}{v^{2}+\omega^{2}}\left\{v\left[\exp \left(v t_{\mu}\right) \cos \left(\omega t_{\mu}\right)-1\right]\right. \\
& \left.+\omega \exp \left(v t_{\mu}\right) \sin \left(\omega t_{\mu}\right)\right\} \\
& +\frac{a \gamma \exp (y)}{\omega}\left[\sin \left(\omega t_{\mu}\right)-\sin \left(\omega t_{e}\right)\right] \\
& +j\left[\frac { a ( 1 + \gamma ) } { u ^ { 2 } + \omega ^ { 2 } } \left\{\omega\left[\exp \left(u t_{e}\right) \cos \left(\omega t_{e}\right)-1\right]\right.\right. \\
& \left.-u \exp \left(u t_{e}\right) \sin \left(\omega t_{e}\right)\right\} \\
& -\frac{a \gamma}{v^{2}+\omega^{2}}\left\{\omega\left[\exp \left(v t_{\mu}\right) \cos \left(\omega t_{\mu}\right)-1\right]\right. \\
& \left.-v \exp \left(v t_{\mu}\right) \sin \left(\omega t_{\mu}\right)\right\} \\
& \left.-\frac{a \gamma \exp (y)}{\omega}\left[\cos \left(\omega t_{e}\right)-\cos \left(\omega t_{\mu}\right)\right]\right]
\end{aligned}
$$

where

$$
a=q v_{e} / w
$$

$$
\begin{gathered}
\gamma=v_{h} / v_{e} \\
u=\alpha v_{e} \\
v=\alpha v_{\mu} \\
y=\alpha w \\
t_{e}=w / v_{e} \\
t_{\mu}=w / v_{\mu} .
\end{gathered}
$$

This function will be displayed graphically in connection with the comparison of analytical and simulation results presented in the next section.

\section{Comparison of Analytical and Simulation RESULTS}

Starting with the carrier transport equations, Riad and Hayes [27] carried out a simulation of both the magnitude and phase of the average frequency response for a singlecarrier CAPD. Inverse Fourier transformation was used to obtain the average impulse response function $\langle h(t)\rangle$ in response to a single photocarrier. Since the conditions assumed in our theoretical model are identical to those assumed for their simulation, it is useful to compare the two results.

The comparison is presented in Figs. 3 and 4. In Fig. 3(a) and (b), the theoretical average impulse response functions $\langle h(t)\rangle$ versus time $t$, calculated from (11), are shown as the solid curves. The simulation results of Riad and Hayes [27] are shown as the dashed curves. Fig. 3(a) represents single-hole injection whereas Fig. 3(b) represents single-electron injection. The theoretical parameter values were chosen to be identical in both cases: electron velocity $v_{e}=10^{7} \mathrm{~cm} / \mathrm{s}$, hole velocity $v_{h}=5 \times 10^{6} \mathrm{~cm} /$ $\mathrm{s}$, and width of multiplication region $w=3 \mu \mathrm{m}$. The holeionization coefficient in Fig. 3(a) is $\beta=5365 \mathrm{~cm}^{-1}$ whereas the electron-ionization coefficient in Fig. 3(b) is $\alpha=5365 \mathrm{~cm}^{-1}$. The ordinates are displayed in units of nanoamperes while the abscissas are in units of picoseconds. The area under each curve is the average gain $\langle M\rangle$ $=5$. Riad and Hayes' results were presented only in normalized form; their results therefore had to be adapted in order to effect a comparison with our absolute calculations. (It should be noted that while their treatment is sufficiently general to allow for the dependence of electric field on position and the dependence of ionization coefficients on field, such relationships were not included in the comparison presented here.)

The frequency response functions are shown in Fig. 4. The solid curves represent the theoretical average frequency response magnitude $(|H(j \omega)|$, left ordinate) and theroretical average frequency response phase $(\angle H(j \omega)$, right ordinate), versus frequency (in gigahertz), as calculated from (13). The dashed and dotted curves represent the same quantities, respectively, obtained from simulation studies by Riad and Hayes [27]. Again, the theoretical parameter values were chosen to be identical in the two cases. The curves in Fig. 4(a) represent single-hole 


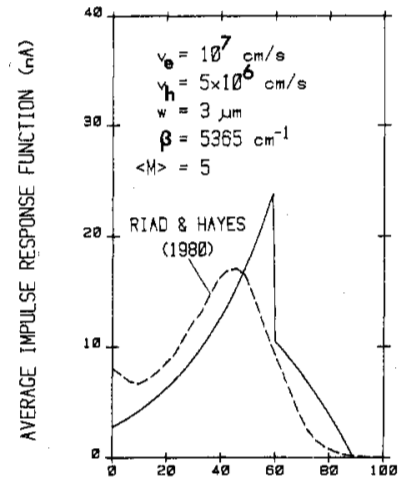

(a)

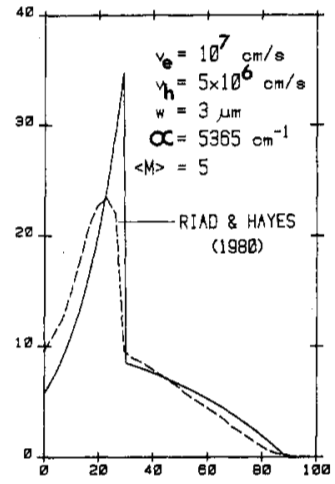

(b)
Fig. 3. Theoretical average impulse response functions $\langle h(t)\rangle$ versus time $t$ (solid curves) calculated from (11) and simulation results of Riad and Hayes [27] (dashed curves). The theoretical parameter values were chosen to be the same as those used by Riad and Hayes: electron velocity $v_{e}=10^{7} \mathrm{~cm} / \mathrm{s}$, hole velocity $v_{h}=5 \times 10^{6} \mathrm{~cm} / \mathrm{s}$, and width of multiplication region $w=3 \mu \mathrm{m}$. The curves in (a) represent single-hole injection with an ionization coefficient $\beta=5365 \mathrm{~cm}^{-1}$ whereas those in (b) represent single-electron injection with an ionization coefficient $\alpha=5365$ $\mathrm{cm}^{-1}$. The ordinates are displayed in units of nanoamperes whereas the abscissas are in units of picoseconds. The area under each curve is the average gain $\langle M\rangle=5$. Dashed curves are adapted from $[27$, Fig. 2(b) and $3(b)]$

injection whereas those in Fig. 4(b) represent single-electron injection. These curves are inverse Fourier transforms of those shown in Fig. 3.

For both the average impulse response functions shown in Fig. 3 and the average frequency response functions displayed in Fig. 4, there is quite good agreement between the analytical and simulation results. It is likely that the somewhat smoother appearance of the simulation curves stems from the windowing associated with the actual computation process. The agreement provides some measure of confirmation that the approach we have used is proper.

\section{Discussion ANd Conclusion}

An analytical expression for the time course of the average impulse response function arising from a single photocarrier entering the multiplication region of a CAPD has been derived, under the assumption of single-carrier-initiated/single-carrier multiplication. The model is applicable to an idealized p-i-n-type single-carrier APD with constant electric field in the avalanche region. With little modification, it should also be useful for devices like the channeling APD [42], [43] in which the carriers are spatially separated and the multiplication is essentially single-carrier-like and continuous. The basis of the calculation was the marked filtered Bernoulli branching process used to study the behavior of the staircase APD [11]. Delta-function absorption was assumed at the boundary of the multiplication region. The avalanche buildup time $\tau_{a v}$ was nil since SCISCM conditions were assumed to prevail. An analytical result for the associated average frequency response function $H(j \omega)$ was derived by Fourier transformation.

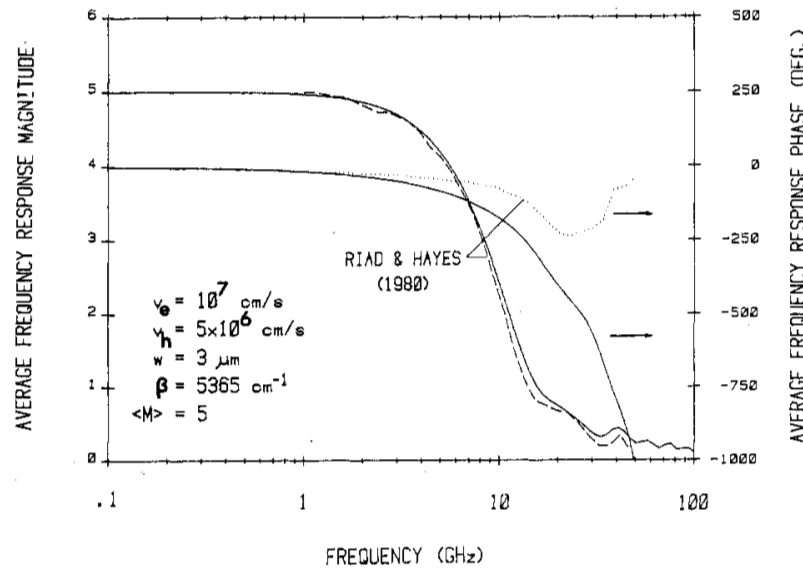

(a)

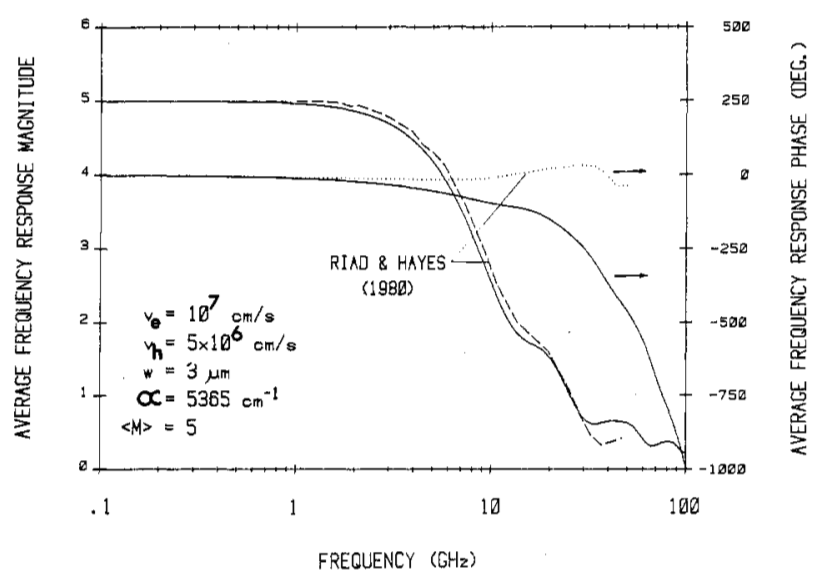

(b)

Fig. 4. Solid curves represent theoretical average frequency response magnitude $(|H(j \omega)|$, left ordinate) and theoretical average frequency response phase ( $\angle H(j \omega)$, right ordinate), versus frequency (in gigahertz), as calculated from (13). Dashed and dotted curves represent the same quantities, respectively, obtained from simulation studies by Riad and Hayes [27]. The theoretical parameter values were chosen to be the same as those used by Riad and Hayes: electron velocity $v_{e}=10^{7} \mathrm{~cm} / \mathrm{s}$, hole velocity $v_{h}=5 \times 10^{6} \mathrm{~cm} / \mathrm{s}$, and width of multiplication region $w=3$ $\mu \mathrm{m}$. The curves in (a) represent single-hole injection with an ionization coefficient $\beta=5365 \mathrm{~cm}^{-1}$ whereas those in (b) represent single-electron injection with an ionization coefficient $\alpha=5365 \mathrm{~cm}^{-1}$. The results in (a) and (b) correspond to the average impulse response functions shown in Fig. 3(a) and (b), respectively. Dashed and dotted curves are adapted from $[27$, Figs. 2(a) and $3(\bar{a})]$.

The analytical results were found to be in good accord with the impulse response functions that Riad and Hayes [27] obtained by simulation from the transport equations. The connection between the branching-process point of view and the transport-equation point of view can be understood as follows. In the limit of an infinite number of stages (continuous branching), the underlying Bernoulli branching process used in our derivation becomes the Yule-Furry branching process [11]-[13], [18]. This is a pure birth process with linear birth rate, which can be described in terms of a stochastic differential equation [44, p. 302, Eq. (5.18)]. With appropriate marking and filtering, this equation becomes the transport equation [26]. Indeed, as has already been pointed out [13], the negative binomial gain distribution derived by McIntyre for a 
CAPD with single-carrier multiplication [5] arises identically from the Yule-Furry process [44].

For the SAGM APD and the RAPD, the motion of carriers in the depletion region outside the multiplication region can contribute substantially to the duration of the impulse response. Thus the multiplication-region impulse response function must be augmented by a contribut on arising from the transit time $\tau_{t r}$. Ruegg [16] derived the single-photocarrier impulse response function for the SCISCM RAPD, assuming exponential light absorption in the nonmultiplying depletion region and equal carrier velocities for electrons and holes. He considered only the current arising from multiplied holes traveling bick through the depletion region, neglecting the contribution of the primary carrier to the loop current. Avalanche buildup time was ignored. Nevertheless, the result that he derived turns out to be quite similar to the contribution from the multiplication region provided in (11).

The results derived here are predicated on the assurr ption of a single photocarrier entering the multiplication region. In the usual experimental situation, a random (Poisson) number of photocarriers is generated. Although the average impulse response function measured in the time domain will accord with our calculations, this is not the case with power-spectral densities which involve second-order statistics. Thus, the kinds of spectral measurements and impulse response function calculations used by Andersson et al. [45] are somewhat different in nature from the quantities we have calculated.

It is worth pointing out that a more realistic theoretical treatment of the CAPD time and frequency response would invoke random, rather than deterministic, carrier transit time. This would result in a smoothing of the theoretical impulse response functions, as was seen for the staircase APD [11, Fig. 11]. Finally, although our enphasis has been on the time response of the single-carrier CAPD, it would be useful to carry out analogous calclilations for double-carrier devices.

\section{ACKNOWLEDGMENT}

We are grateful to R. J. McIntyre of RCA and G. l' Williams of AT\&T Bell Laboratories for many useful discussions.

\section{REFERENCES}

[1] S. D. Personick, Fiber Optics. New York: Plenum, 1985.

[2] R. J. McIntyre, "Multiplication noise in uniform avalanche diodes:" IEEE Trans. Electron Devices, vol. ED-13, pp. 164-168, 1966.

[3] S. D. Personick, "New results on avalanche multiplication statistiss with application to optical detection," Bell Syst. Tech. J., vol. 53, pp. 167-189, 1971.

[4] - "Statistics of a general class of avalanche detectors with app. ications to optical communication," Bell Syst. Tech. J., vol. 50, ps. 3075-3095, 1971.

[5] R. J. McIntyre, "The distribution of gains in uniformly multiplying avalanche photodiodes: Theory," IEEE Trans. Electron Devices, vcl. ED-19, pp. 703-713, 1972.

[6] J. Conradi, "The distribution of gains in uniformly multiplying av ilanche photodiodes: Experimental," IEEE Trans. Electron Device., vol. ED-19, pp. 713-718, 1972.

[7] P. P. Webb, R. J. McIntyre, and J. Conradi, "Properties of avillanche photodiodes," $R C A$ Rev., vol. 35, pp. 234-278, 1974.
[8] P. P. Webb and R. J. McIntyre, "Recent developments in silicon avalanche photodiodes," RCA Eng., vol. 27, pp. 96-102, 1982.

[9] F. Capasso, W. T. Tsang, and G. F. Williams, "Staircase solid-state photomultipliers and avalanche photodiodes with enhanced ionization rates ratio," IEEE Trans. Electron Devices, vol. ED-30, pp. 381$390,1983$.

[10] B. L. Kasper, J. C. Campbell, and A. G. Dentai, "Measurements of the statistics of excess noise in separate absorption, grading and multiplication (SAGM) avalanche photodiodes," Electron. Lett., vol. 20, pp. 796-798, 1984.

[11] K. Matsuo, M. C. Teich, and B. E. A. Saleh, "Noise properties and time response of the staircase avalanche photodiode," IEEE Trans. Electron Devices, vol. ED-32, pp. 2615-2623, 1985; also in J. Lightwave Technol, vol. LT-3, pp. 1223-1231, 1985.

[12] M. C. Teich, K. Matsuo, and B. E. A. Saleh, "Excess noise factors for conventional and superlattice avalanche photodiodes and photomultiplier tubes," IEEE J. Quantum Electron., vol. QE-22, pp. 1184$1193,1986$.

[13] M. C. Teich, K. Matsuo, and B. E. A. Saleh, "Counting distributions and error probabilities for optical receivers incorporating superlattice avalanche photodiodes," IEEE Trans. Electron Devices, this issue, pp. $1475-14 \overline{8}, 1986$.

[14] W. T. Read, "A proposed high frequency negative resistance diode," Bell Syst. Tech. J., vol. 37, pp. 401-446, 1958.

[15] T. Kaneda, H. Takanashi, H. Matsumoto, and T. Yamaoka, "Avalanche buildup time of silicon reach-through photodiodes," J. Appl. Phys., vol. 47, pp. 4960-4963, 1976.

[16] H. W. Ruegg, "An optimized avalanche photodiode," IEEE Trans. Electron Devices, vol. ED-14, pp. 239-251, 1967.

[17] J. C. Campbell, A. G. Dentai, W. S. Holden, and B. L. Kasper, "High-performance avalanche photodiode with separate absorption 'grading' and multiplication regions,'" Electron. Lett., vol. 19, pp. 818-820, 1983.

[18] K. Matsuo, M. C. Teich, and B. E. A. Saleh, "Poisson branching point processes," J. Math. Phys., vol. 25, pp. 2174-2185, 1984.

[19] R. B. Emmons and G. Lucovsky, "The frequency response of avalanching photodiodes," IEEE Trans. Electron Devices, vol. ED-13, pp. 297-305, 1966.

[20] R. B. Emmons, "Avalanche-photodiode frequency response," $J$. Appl. Phys., vol. 38, pp. 3705-3714, 1967.

[21] J. J. Chang, "Frequency response of PIN avalanching photodiodes," IEEE Trans. Electron Devices, vol. ED-14, pp. 139-145, 1967.

[22] C. A. Lee, R. L. Batdorf, W. Wiegmann, and G. Kaminsky, "Time dependence of avalanche processes in silicon," J. Appl. Phys., vol. 38, pp. 2787-2796, 1967.

[23] I. Naqvi, "Effects of time dependence of multiplication process on avalanche noise,': Solid-State Electron., vol. 16, pp. 19-28, 1973.

[24] S. Rakshit and R. Sarin, "Frequency responses of graded-bandgap low-noise avalanche photodiodes," IEEE Trans. Electron Devices, vol. ED-32, pp. 749-752, 1985.

[25] S. M. Riad and A. A. R. Riad, "Time-domain simulation analysis of avalanche photodetectors," IEEE Trans. Electron Devices, vol. ED29, pp. 994-998, 1982.

[26] A. A. Walma and R. Hackam, "On the time dependency of the avalanche process in semiconductors," Solid-State Electron., vol. 18, pp. 511-517, 1975.

[27] A. A. R. Riad and R. E. Hayes, "Simulation studies in both the frequency and time domains of InGaAsP-InP avalanche photodetectors," IEEE Trans. Electron Devices, vol. ED-27, pp. 1000-1003, 1980.

[28] G. F. Williams, F. Capasso, and W. T. Tsang, "The graded bandgap multilayer avalanche photodiode: A new low-noise detector," IEEE Electron Device Lett., vol. EDL-3, pp. 71-73, 1982.

[29] K. Brennan, "Theory of electron and hole impact ionization in quantum well and staircase superlattice avalanche photodiode structures," IEEE Trans. Electron Devices, vol. ED-32, pp. 2197-2205, 1985.

[30] G. E. Stillman, V. M. Robbins, and N. Tabatabaie, "III-V compound semiconductor devices: Optical detectors," IEEE Trans. Electron Devices, vol. ED-31, pp. 1643-1655, 1984.

[31] F. Capasso, "Physics of avalanche photodiodes," in Semiconductors and Semimetals, R. K. Willardson and A. C. Beer, Series Eds., vol. 22, part D, Lightwave Communications Technology, W. T. Tsang, Ed. New York: Academic, 1985, pp. 1-172.

[32] R. Chin, N. Holonyak, G. E. Stillman, J. Y. Tang, and K. Hess, "Impact ionization in multilayered heterojunction structures,"' Electron. Lett., vol, 16, pp. 467-469, 1980. 
[33] F. Capasso, W. T. Tsang, A. L. Hutchinson, and G. F. Williams, "Enhancement of electron impact ionization in a superlattice: A new avalanche photodiode with a large ionization rate ratio," Appl. Phys. Lett., vol. 40, pp. 38-40, 1982.

[34] K. Mohammed, F. Capasso, J. Allam, A. Y. Cho, and A. L. Hutchinson, "New high-speed long-wavelength $\mathrm{Al}_{0.48} \mathrm{In}_{0 .}{ }_{52} \mathrm{As}$. $\mathrm{Ga}_{0.47} \mathrm{In}_{0.53}$ As multiquantum well avalanche photodiodes," Appl. Phys. Lett., vol. 47, pp. 597-599, 1985.

[35] F.-Y. Juang, U. Das, Y. Nashimoto, and P. K. Bhattacharya, "Electron and hole impact ionization coefficients in $\mathrm{GaAs}-\mathrm{Al}_{x} \mathrm{Ga}_{1-x} \mathrm{As}$ superlattices," Appl. Phys. Lett., vol. 47, pp. 972-974, 1985.

[36] H. Blauvelt, S. Margalit, and A. Yariv, "Single-carrier-type dominated impact ionization in multilayer structures," Electron. Lett., vol. 18, pp. 375-376, 1982.

[37] K. Brennan, "Theory of the GaInAs/AlInAs doped-quantum-well APD: A new low-noise solid-state photodetector for lightwave communications systems," to be published.

[38] J. S. Smith, L. C. Chiu, S. Margalit, A. Yariv, and A. Y. Cho, "A new infrared detector using electron emission from multiple quantum wells," J. Vac. Sci. Technol. B, vol. 1, pp. 376-378, 1983.

[39] S. L. Chuang and K. Hess, "Impact ionization across the conductionband-edge discontinuity of quantum-well heterostructures," J. Appl. Phys., vol. 59, pp. 2885-2894, 1986.

[40] F. Capasso, J. Allam, A. Y. Cho, K. Mohammed, R. J. Malik, A. L. Hutchinson, and D. Sivco, "New avalanche multiplication phenomenon in quantum well superlattices: Evidence of impact ionization across the band-edge discontinuity," Appl. Phys. Lett., vol. 48 , pp. 1294-1296, 1986.

[41] F. Capasso, AT\&T Bell Laboratories, Murray Hill, NJ, private communication.

[42] F. Capasso, "The channeling avalanche photodiode: A novel ultralow-noise interdigitated p-n junction detector," IEEE Trans. Electron Devices, vol. ED-29, pp. 1388-1395, 1982.

[43] K. Brennan, "Theory of the channeling avalanche photodiode," IEEE Trans. Electron Devices, vol. ED-32, pp. 2467-2478, 1985.

[44] E. Parzen, Stochastic Processes. San Francisco: Holden-Day, 1962.

[45] T. Andersson, A. R. Johnston, and H. Eklund, "Temporal and frequency response of avalanche photodiodes from noise measurements," Appl. Opt., vol. 19, pp. 3496-3499, 1980.

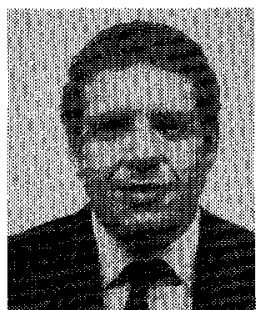

Malvin C. Teich (S'62-M'66-SM'72) was born in New York, NY. He received the S.B. degree in physics from the Massachusetts Institute of Technology, Cambridge, in 1961, the M.S. degree in electrical engineering from Stanford University, Stanford, CA, in 1962, and the Ph.D. degree in quantum electronics from Cornell University, Ithaca, NY, in 1966.

In 1966, he joined the MIT Lincoln Laboratory, Lexington, MA, where he was engaged in work on coherent infrared detection. In 1967, he became a member of the faculty in the Department of Electrical Engineering, Columbia University, New York, NY, where he is now teaching and pursuing his research interests in the areas of optical and infrared detection, quantum optics, lightwave communications, and sensory perception. He served as Chairman of the Department from 1978 to 1980 . He is also a member of the faculty in the Department of Applied Physics and Nuclear Engineering, and a member of the Columbia Radiation Laboratory, the
Center for Telecommunications Research, and the Columbia Bioengineering Institute. He has authored or coauthored some 100 technical publications and holds one patent.

Dr. Teich is a member of Sigma Xi, the American Physical Society, the Acoustical Society of America, the Society for Neuroscience, the American Association for the Advancement of Science, and the New York Academy of Sciences. He served as a member of the Editorial Advisory Panel for Optics Letters from 1977 to 1979 . In 1969 he was the recipient of the IEEE Browder J. Thompson Memorial Prize for his paper "Infrared Heterodyne Detection"' and in 1981 he received the Citation Classic Award of Current Contents for this work. He was appointed a Fellow of the John Simon Guggenheim Memorial Foundation in 1973 and was elected a Fellow of the Optical Society of America in 1983.

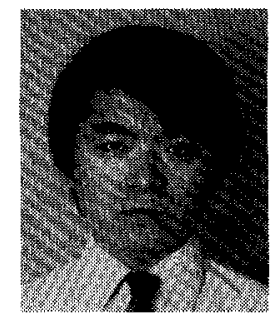

Kuniaki Matsuo (M'85) was born in Hiroshima, Japan. He received the B.S. degree in electrical engineering from Tokyo Electrical Engineering College, Tokyo, in 1972 and the M.S. and Ph.D. degrees in electrical engineering from Columbia University, New York, NY, in 1978 and 1984, respectively.

From 1984 to 1986 he was a Postdoctoral Research Scientist in the Columbia Radiation Laboratory, Department of Electrical Engineering, Columbia University. In 1986, he joined the Hiroshima-Denki Institute of Technology, Hiroshima, Japan, where he is now teaching and pursuing his research interests in avalanche photodetection, quantum electronics, and point processes.

Dr. Matsuo is a member of Sigma Xi.

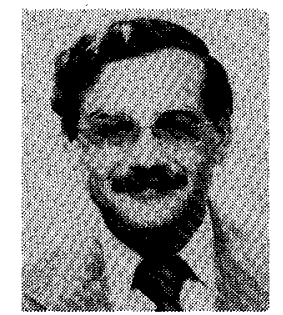

Bahaa E. A. Saleh (M'73-SM'86) received the B.S. degree from Cairo University in 1966 and the Ph.D. degree from the Johns Hopkins University in 1971 , both in electrical engineering.

From 1971 to 1974 he was an Assistant Professor at the University of Santa Catarina, Brazil. Thereafter, he joined the Max Planck Institute in Göttingen, Germany, where he was involved in research in laser light scattering and photon correlation spectroscopy. He is presently Professor of Electrical and Computer Engineering at the University of Wisconsin, Madison, where he has been since 1977. He held visiting appointments at the University of California, Berkeley, in 1977, and the Columbia Radiation Laboratory of Columbia University in 1983. $\mathrm{He}$ is currently involved in research in image processing, optical information processing, statistical optics, optical communication, and vision. $\mathrm{He}$ is the author of Photoelectron Statistics (Springer, 1978) and a co-editor of Transformations in Optical Signal Processing (SPIE, 1981). In 19801983, he was an associate editor of the Journal of the Optical Society of America, and since 1983 he has been a topical editor of the same journal.

Dr. Saleh is a Fellow of the Optical Society of America and a member of Phi Beta Kappa and Sigma Xi. He was appointed a Fellow of the John Simon Guggenheim Foundation in 1984. 